\title{
Observational properties of the open cluster system of the Milky Way and what they tell us about our Galaxy
}

\author{
André Moitinho \\ SIM/FCUL, Faculdade de Ciências da Universidade de Lisboa, Ed. C8, Campo Grande, \\ 1749-016 Lisbon, Portugal \\ email: andre@sim.ul.pt
}

\begin{abstract}
Almost 80 years have passed since Trumpler's analysis of the Galactic open cluster system laid one of the main foundations for understanding the nature and structure of the Milky Way. Since then, the open cluster system has been recognised as a key source of information for addressing a wide range of questions about the structure and evolution of our Galaxy. Over the last decade, surveys and individual observations from the ground and space have led to an explosion of astrometric, kinematic and multiwavelength photometric and spectroscopic open cluster data. In addition, a growing fraction of these data is often time-resolved. Together with increasing computing power and developments in classification techniques, the open cluster system reveals an increasingly clearer and more complete picture of our Galaxy. In this contribution, I review the observational properties of the Milky Way's open cluster system. I discuss what they can and cannot teach us now and in the near future about several topics such as the Galaxy's spiral structure and dynamics, chemical evolution, large-scale star formation, stellar populations and more.
\end{abstract}

Keywords. catalogs, ISM: extinction, Galaxy: evolution, Galaxy: disk, open clusters and associations: general

\section{Preamble}

In 1930, Robert Trumpler published his seminal paper in which he proves the existence of the interstellar medium (ISM). In that paper, Trumpler showed how distances to open clusters (OCs) derived from the apparent magnitudes of cluster stars of known spectral types are systematically greater than those derived from their apparent sizes, and how the effect increases with distance. He correctly attributed this phenomenon to the presence of an intervening medium. This was the first paper using OCs as tracers of Galactic structure.

\section{Overview}

Since the work of Trumpler, the open cluster system has been used to characterise, study and analyse a variety of aspects related to the structure, composition, dynamics, formation and evolution of the Milky Way. Not being exhaustive, these aspects include:

- Spatial scales: scale height of $\operatorname{disk}(\mathrm{s})$, height of the Sun above the Galactic plane, distance to the Galactic Centre.

- Spiral structure: tracing spiral arms.

- Kinematics and dynamics: rotation of spiral pattern, Galactic rotation curve (velocity of the 'local standard of rest', Oort constants), orbits of OCs and their relation to cluster survival/disruption. 
- Galactic formation and evolution: ages of the oldest clusters, abundance pattern, inflow rate.

- Distribution (absorption) and kind (extinction law) of interstellar matter.

The potential of using OCs for studying the Milky Way is well known and sentences like "open clusters are valuable objects for Galactic studies" appear in the introduction of almost every OC paper. Leaving aside the astrophysical reasons, the main advantage of using OCs is the precision with which one can derive their reddening values, distances, ages, velocities and metallicities, compared to individual stars. This arises not only from improved statistics, as OCs are ensembles of stars, but also from the distribution of cluster stars in photometric diagrams. In fact, the colour-magnitude and two-colour diagrams (CMDs and TCDs, respectively) of star clusters can exhibit a rich structure that provides bench marks to constrain those parameters beyond a simple statistical average. An example is the 'elbow' displayed in optical CMDs for early A-type stars, which is very useful for constraining distances.

There are also some disadvantages, or at least difficulties and systematics in studying the Galaxy on the basis of OCs. Confusion against crowded backgrounds in the Galactic plane and dissolution of OCs likely produce selection effects that conspire against the identification of faint and/or poorly populated clusters. These effects will produce a selection against old clusters, which are fainter and potentially poorer (due to dynamical evolution) than their younger counterparts, and against the low-mass end of the OC population. The extent of such a selection is far from well known, but it must be kept in mind when deriving conclusions from the observed global properties of the OC system.

Using OCs for studying the Galaxy relies on a huge amount of painstaking data gathering. Each cluster is just a point in parameter space, but requires measuring many stars. Traditionally, stars were measured one by one using photoelectric photometers and spectrographs. Modern CCDs, infrared arrays and multi-object spectrographs (MOSs) have the ability of observing hundreds or thousands of stars in one shot, but reducing the data has proved to be very time consuming. In the last few years, increased computing power and development of automated or semi-automated reduction procedures have alleviated the reduction burden. But it took several years for the community to master software like DAOPHOT (Stetson et al. 1990). I guess that many of us have known what it is to reduce images one by one, selecting by hand the adequate PSF stars, testing the PSF model and redoing it if necessary. In the mid 1990s, the workstation I had at work would take several hours just to run ALLSTAR on a $2048 \times 2048$-pixel frame. Now, this can be done in a few minutes, or less, and most of us have used experience to devise automatic ways to select the proper PSF stars, so that a whole observing run can be reduced fairly quickly.

The actual job of studying the Galaxy requires a catalogue of OC parameters (reddening values, distances, ages, velocities, metallicities, diameters). Thousands of studies of individual OCs exist in the literature, often with nonconcordant results which must be critically inspected and collated. Despite the care put into the process, the variety of techniques used by different authors to observe, reduce and derive parameters, lead to inhomogeneous compilations that can limit their usefulness. This problem is well known and has motivated several attempts of deriving homogeneous parameter sets, usually by adopting some well-defined analysis procedure, reference curves (e.g., the zero-age main sequence, ZAMS, and isochrones) and measurements. Still, the problem persists for most objects.

It is therefore clear that assembling the observational basis for studying the Galaxy is an incremental process. It is built up slowly by adding the results of individual cluster studies, each one contributing one probing point. It is therefore natural that qualitative 
leaps in our understanding of the Galaxy happen in intervals of many years. Roughly, such jumps take about 20 years.

Very schematically, in the 1980s there was the Lund catalogue (Lyngå 1987), which contained around 1200 clusters of which a few hundred had some of their parameters determined. The classic review on OCs and Galactic structure is that of Janes \& Adler (1982) and its update (Janes et al. 1988). The 1990s brought us the on-line WEBDA OC database (formerly BDA; Mermilliod 1992) which not only included a list of clusters and their parameters (essentially the Lund catalogue), but also the actual measurements of their stars (photometry, astrometry, spectral types, etc.). From the observational point of view, CCDs and infrared arrays became of common use, Hipparcos provided a wealth of astrometric and kinematic data and settled the distance scale through accurate parallaxes of the Hyades and Pleiades. Although the new data did not bring in new significant results regarding the spatial structure of the Milky Way, the number of abundance measurements and discovery of more old OCs shifted the focus on the origin and chemical evolution of the Galaxy. The canonical review on the subject is that of Friel (1995) and its updates. The 2000s brought the Dias et al. (2002) catalogue of OCs, which updates the Lund catalogue. The latest version includes 1787 clusters and has eliminated a number of entries shown to be nonexistent. The 2MASs survey (Cutrie et al. 2003) has triggered searches of new clusters (optically detectable and embedded, e.g., Bica et al. 2003b; Dutra et al. 2003; Froebrich et al. 2007) but very few of them have actually been studied. So far, this decade has not yet provided a fresh review on our subject, although some refined recalculations taking advantage of currently available data have been published (Bonatto et al. 2006; Piskunov et al. 2006). However, the rapidly growing number of spectroscopic observations allowed by MOSs, availability of reduction pipelines and ongoing large-scale photometric surveys are expected to soon take the understanding of our Galaxy to the next step.

\section{The sample}

As discussed above, knowledge of OCs is compiled in catalogues. The most complete catalogue of optically revealed OCs is that of Dias et al. (2002) †. Its latest version (2.10) lists 1784 objects of which 969 (54\%) have simultaneous reddening, distance and age determinations. The number of clusters with distance, ages, radial velocities and proper motions is much smaller (430, or $24 \%$ ) and only $178(10 \%)$ have their metallicities determined. Lists of infrared (IR) OCs and candidates have been compiled by Bica et al. (2003a,b), Dutra et al. (2003) and Froebrich et al. (2007), based on searches using 2MASS data products. These objects, detected in the IR, add about 700 clusters to the optically identified family. However, very few have been studied so far and many might not be real OCs. The main references for the IR cluster lists are Bica et al. (2003a,b) and Dutra et al. (2003). The systematic IR search of Froebrich et al. (2007) has produced a list of around 1000 candidates, which they estimate suffers from $50 \%$ false-detection contamination. Once considering the number of already identified IR clusters, the Froebrich et al. (2007) list still contributes with the discovery of about 150 OCs (Glushkova et al. 2009).

The distribution of optical and IR clusters is shown in Figure 1. It is noticeable how the IR clusters are mostly located close to the $b=0^{\circ}$ plane, while the optical clusters define a thicker disk. This is expected, since most obscuring material is defining what is known as the extreme thin disk. The number distribution of clusters is not uniform along

$\dagger$ http://www.astro.iag.usp.br/ ${ }^{\sim}$ wilton/ 


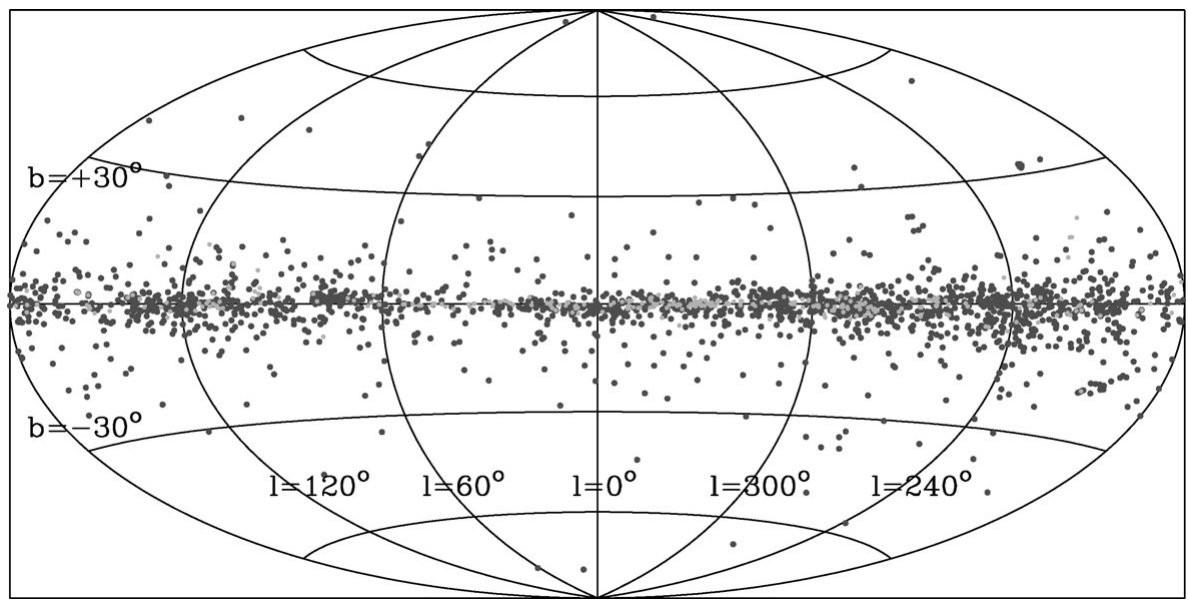

Figure 1. Aitoff distribution of known open clusters and candidates on the Galactic plane. Black: optically visible clusters. Grey: infrared clusters.

the Galactic longitude range. Moreover, clusters in different age groups exhibit different distributions (e.g., figure 3 of Bonatto et al. 2006).

At least two obvious biases modulate the distribution of identified clusters. The first is introduced by the extinction produced by dense interstellar material. Figure 2 clearly shows how the OCs delineate the wavy dust disposition. That extinction affects the detectability of sources is well known, but the kind of patterns it introduces in the distribution of OCs is often overlooked in studies of the Galaxy using OCs (and other objects). The other bias is produced by crowded backgrounds, against which an OC might contribute little to the density of field stars and can thus remain unidentified. This effect also includes nearby sparse OCs that occupy large areas on the sky.

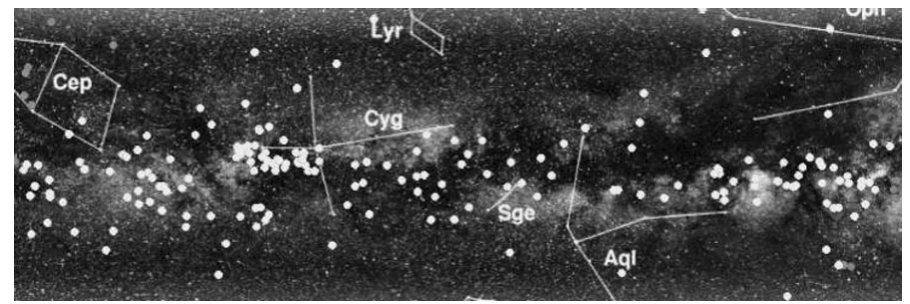

Figure 2. Optically visible OCs (circles) plotted over an optical image of the first quadrant of the Milky Way (credit A. Mellinger).

But there are also biases in the sample of studied clusters. On the one hand, interest has focussed on studying specific regions of the Galaxy (e.g., Cygnus, Carina and PuppisVela), thus artificially increasing the number of studied clusters in those regions with respect to others. On the other hand, many clusters have been observed for reasons other than studying the Galaxy. This was especially true in the past, when focus was mostly on studying stellar evolution. The tendency has been to study bright and/or well-populated clusters, which would provide much clearer Hertzsprung-Russell diagrams. Given the aforementioned difficulties in data reduction, there was little motivation to study poorer (potentially older) and fainter (potentially older/more distant/more reddened).

Although these biases exist in principle, all-sky proper-motion and photometric surveys can help to overcome them. The Hipparcos/Tycho-2 (ESA 1997; Høg et al. 2000) 
and UCAC2 (Zacharias et al. 2004) astrometric (and photometric) catalogues, the 2MASS $J H K$ photometry and images, as well as other available data have enabled identification of OCs beyond simple visual recognition. Searches for concentrations of stars at a common distance or with common proper motion, cluster sequences in CMDs or simply spatial concentrations of stars obeying some photometric criteria have allowed discovery of more than a hundred optically visible OCs (e.g., Platais et al. 1998; Alessi et al. 2003; Kharchenko et al. 2005) and a few hundred IR clusters, as mentioned above.

$200 \mathrm{Myr}, 1,2,3 \mathrm{Gyr}-$ no extinction

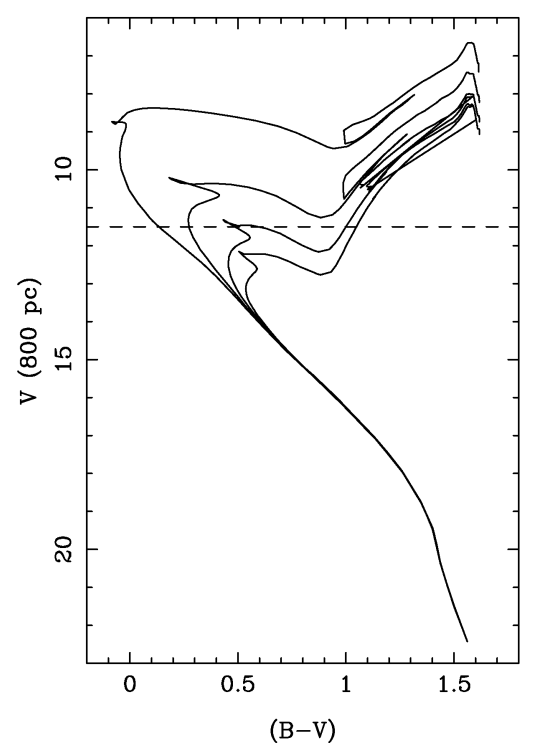

Figure 3. 0.2, 1, 2 and 3 Gyr isochrones (Girardi et al. 2000) at a distance of 800 pc from the Sun. No extinction is applied. The dashed line indicates the $11.5 \mathrm{mag}$ completeness limit of the stellar data catalogue used in Piskunov et al. (2006).

It has been argued that the sample of known OCs is complete within 850 pc (Piskunov et al. 2006; $1 \mathrm{kpc}$ is also quoted). The argument is based on the fact that the number of clusters $\mathrm{kpc}^{-1}$ starts to decrease beyond $850 \mathrm{pc}$. It seems, however, that even within $850 \mathrm{pc}$ the sample of OCs might be missing a significant number of poorly populated intermediate-age and old objects. Figure 3 shows a series of isochrones, from moderately young to moderately old, placed at 800 pc from the Sun, with no extinction applied. It is readily seen that at 1 Gyr and older, only the evolved portion of the cluster sequence is above the dashed line, which marks the completeness limit of the astrometric and photometric catalogue used in Piskunov et al. (2006). Apart from the red clump, the other post-main-sequence regions of the CMD will be barely populated. The red clump will only be noticeable for fairly populated clusters. For the not-so-rich clusters, the number of clump stars might not be enough to produce a visible cluster signature either in proper-motion vector-point diagrams or in CMDs. The ability to identify a cluster based on kinematics will also be hampered along directions dominated by the solar motion. Furthermore, in reality, clusters will be affected by extinction, which has the effect of making the sequences fainter, thus pushing them even further below the completeness line. For the 200 Myr example, the red clump is very sparsely populated and the presence of 1 mag of absorption will leave the out the main sequence, thus making it very hard to detect (unless it is visually obvious on a sky chart). 
As for the total number of OCs in the Milky Way, estimates depend on many hypotheses, such as how well the number of clusters per volume in the solar neighbourhood represents its position relative to the Galactic Centre, the density-decay law and cluster completeness. In any case, estimates indicate numbers on the order of $10^{5} \mathrm{OCs}$ (Bonatto et al. 2006; Piskunov et al. 2006).

\section{Spatial scales}

We now proceed to see what the statistical properties of the OC distribution tell us about the spatial scales of the Galactic disk and our position within it. The increased samples of OCs analysed in recent studies essentially recover the classic results from the 1980s, although in a clearer way. In particular, it is found that the disk scale height (SH) defined by the young clusters is smaller than that derived from older clusters, with the young and intermediate-age OCs ( $<200$ Myr versus $<1$ Gyr) having SHs of 48 pc and $\sim 150$ pc, respectively. For older clusters, the vertical distribution is essentially uniform, so that no SH can be derived. Also, the distribution of OCs younger than $\sim 1$ Gyr clearly shows how the disk outside the solar circle is $1.4-2.0 \times$ wider than inside this radius (Bonatto et al. 2006). Assuming that OCs are symmetrically distributed above and below the plane, the zero point of the height distribution gives a measure of the distance of the Sun from the Galactic plane. The current values determined from OCs range from about 15 to 22 pc (Bonatto et al. 2006; Piskunov et al. 2006; Joshi 2007).

These seem to be well-established properties of the OC system that persist as the sample grows. Interpretation is, however, not so straightforward and unambiguous. One of the first things that call our attention is that the SH defined by thin-disk stars $(\sim 200 \mathrm{pc})$ is much larger that that defined by the young OCs, although it is more or less compatible with that of intermediate-age OCs. This could be seen as a disk evolutionary effect, by which the disk would thicken with age due to some heating mechanism such as collisions with external systems or by different conditions in the ancient disk (Janes \& Phelps 1994). However, it could also be an effect of the selective destruction of OCs at lower heights. Indeed, the range of heights of the different $\mathrm{OC}$ age groups are very similar. What is different is the proportion of clusters at small to large heights (see, e.g., figure 7 of Bonatto et al. 2006). This might as well be simply reflecting the destruction of clusters that tend to be close to the plane, which would trim the distribution peak, leading to a flatter distribution and hence a larger SH. This scenario seems consistent with the apparent flatness of the old OC distribution, which is not seen in the smaller sample of Janes \& Phelps (1994), who find a SH of $\sim 375$ pc. Verification requires a kinematic analysis.

From the perspective of OCs as progenitors of the field population, the link between the OC and field-star SHs remains to be firmly established. Kroupa (2002) has shown how (kinematically hot) stars escaping star clusters, mostly while emerging from their parental molecular clouds, can thicken Galactic disks and even produce thick disks. This mechanism could provide the link. Another interesting consequence is that if this mechanism were the sole avenue for producing a thick disk, then we would not expect to find thick-disk OCs. Finding these clusters would mean that other processes (such as capture from an accreted galaxy) would (also) operate.

As for the the thicker SH outside the solar circle, the result is derived from a global comparison of the SH of outer versus inner clusters. Its precise meaning is not straightforward and is most likely a combination of effects such as the flaring of the disk and the amplitude of the warp. Isolating both effects requires larger samples of OCs to analyse the $\mathrm{SH}$ at smaller Galactic longitude intervals. 
OCs have also been used for estimating the distance to the Galactic Centre. From a kinematic analysis of 301 OCs within $3 \mathrm{kpc}$ from the Sun, Shen \& Zhu (2007) derive $R_{\mathrm{GC}} \sim 7.95 \mathrm{kpc}$, which is consistent with the the value the same authors derive from OB stars $(8.25 \mathrm{kpc})$. Although based on a simple Oort model, these determinations agree with the well-known results of Genzel et al. $(2000 ; 7.8-8.2 \mathrm{kpc})$ from analysis of radial velocities of more than 100 stars around the black hole Sgr A*, and of Eisenhauer et al. (2003), who found $R_{\mathrm{GC}}=7.94 \pm 0.42 \mathrm{kpc}$ based on astrometric and radial-velocity observations of star S2 around Sgr A*. These results support the long-known indications that the standard IAU value, $R_{\mathrm{GC}} \equiv 8.5 \mathrm{kpc}$, should be revised and lowered by $\sim 500 \mathrm{pc}$. A synthesis of the determinations of $R_{\mathrm{GC}}$ in the past decades is shown in figure 2 of Shen \& Zhu (2007).

Another aspect concerning the spatial scales of the Galactic disk is the extent of the warp. By looking along low-extinction windows in the third Galactic quadrant (3GQ), Vázquez et al. (2008) have been able use OCs to trace the disk out to Galactocentric distances greater than $16 \mathrm{kpc}$. In their figure 6, one can see how the southern warp reaches distances greater than $1 \mathrm{kpc}$ below the formal Galactic plane. Finally, Carraro et al. (2009) reported OCs at large Galactocentric distances, with VdB-Hagen 4 reaching $20 \mathrm{kpc}$. This value is significantly larger than the $\sim 14 \mathrm{kpc}$ truncation limit usually adopted and sets a lower limit to the extent of the stellar disk as probed by OCs.

\section{Spiral structure}

Young OCs have long been used for tracing spiral structure (e.g., Becker \& Fenkart 1970; Moffat et al. 1979). Since star formation in the Milky Way occurs mainly in the spiral arms, it is expected that the younger clusters, which have not yet departed significantly from their birth sites, will trace the local spiral structure. Compared to gaseous spiral indicators such as $\mathrm{HI}$, HiI and CO, which are observed at radio wavelengths and for which we rely on rotation curves to derive their distances, OC distances are much better determined. Furthermore, it is well known that the velocity field in spiral arms does not correspond exactly to that implied by the general rotation curve. Indeed, spiral arms appear as glitches in empirical rotation curves. Thus, one arrives at the situation of tracing spiral structure using velocity-distance relations that do not apply well to the regions where they are used. Despite this source of uncertainty, radio observations roughly reveal the general spiral structure of the Milky Way, with the advantage of being insensitive to obscuration and thus being able to reach most of the Galaxy. OC distances are more precise, but are mainly obtained from observations in the optical domain and cannot probe beyond a few kpc due to interstellar obscuration (except for a few absorption windows at certain Galactic longitudes).

When mentioning young OCs as spiral tracers, the question arises as to what is meant by young. Dias \& Lépine (2005), in their figure 1, show that OCs up to $\sim 12$ Myr remain in their parent arms. At $\sim 20 \mathrm{Myr}$, they are already drifting away from the arms, joining the general disk population, although spiral traces are still identifiable. This figure is illustrative of spiral structure as traced by OCs and also shown in many other studies. In particular, the Carina-Sagittarius, local (or Orion) and Perseus arms are prominent in the solar vicinity up to distances of $\sim 4-5 \mathrm{kpc}$.

Expanding on this classical view, the recent papers of Carraro et al. (2005), Moitinho et al. (2006) and Vázquez et al. (2008) have revealed the spiral structure in the 3GQ based on OCs, CO clouds and early-type field stars. These studies support a four-armed pattern and have shown that the Outer (Norma-Cygnus) arm crosses the whole 3GQ, reaching distances beyond $10 \mathrm{kpc}$ from the Sun and that the local (Orion) arm is more 
than a simple spur, reaching the Outer arm. As for Perseus, it is not traced by young OCs in this region, being apparently disrupted by the crossing of the local arm, but is then again traced by OCs in the 4GQ. An aspect of these analyses that is worthwhile emphasising is the treatment of early-type field stars. At sufficiently large distances, early-type stars belonging to spiral arms can be considered as spatially confined along their lines of sight. These stars will thus produce cluster-like sequences in photometric diagrams that allow determining average reddening values and distances using the same techniques as used for analysing clusters. Interestingly, these sequences had previously been identified and baptised as 'blue plumes' (because of their plume-like appearance on the blue side of CMDs) and were interpreted as the signature of a dwarf galaxy cannibalised by the Milky Way (the Canis Major dwarf galaxy; Martínez-Delgado et al. 2005). However, these blue plumes have been shown not only to trace the spiral structure in the 3GQ, but also across the 2GQ (Carraro et al. 2008), where due to superposition with evolved OCs they have often been interpreted as blue stragglers.

\section{Kinematics and dynamics}

The 1990s and 2000s witnessed an explosion of astrometric data. The main players being the Hipparcos and Tycho-2 catalogues (ESA 1997; Høg et al. 2000) and the UCAC2 compilation (Zacharias et al. 2004). The new UCAC3 catalogue is being released during this IAU General Assembly.

These data have led to many studies of OCs. From the individual cluster point of view, they have allowed to determine cluster membership probabilities, cluster proper motions, internal dynamics and to firmly set the Hyades and Pleiades luminosity scales, which are crucial for calibrating the cosmological distance ladder and the ages provided by stellar evolution models. These astrometric data have also been widely used for studying starforming regions (which are out of the scope of this review) containing OCs. But regarding large-scale Galactic structure, they have not triggered great qualitative progress. The main reason for this is the very limited distance range, of a few hundred pc, that they can probe.

OCs have often been used for studying the local behaviour of the Galactic rotation curve. However, a proper rotation curve covering a sizable range of the Galaxy is still lacking. Work in progress in this area was recently reported by Frinchaboy \& Majewski (2008). The outcome of most kinematic research has been to improve the determination of parameters such as the local standard of rest rotation speed, Oort's constants and the velocity of the Sun. An example of such an analysis is summarised in table 2 of Piskunov et al. (2006). These improvements do not only come from the available highquality astrometric data, but also from the quantity of data, which allows to resolve local structures such as star-formation complexes and stellar streams and eliminate the systematics they introduce (e.g., Piskunov et al. 2006).

In addition to these more classical calculations, OCs have also been used recently to determine the rotation speed of the spiral pattern. Dias \& Lépine (2005) confirmed that spiral arms rotate as rigid bodies, and determined the pattern speed by direct integration of cluster orbits and by comparing the positions of clusters in different age groups, leading to an adopted value of $24 \mathrm{~km} \mathrm{~s}^{-1} \mathrm{kpc}^{-1}$. As a an additional result, they find that the corotation radius is $(1.06 \pm 0.08) \times$ the solar Galactocentric distance. It is also interesting to note that Bobylev et al. (2007) find a different speed for the Orion arm compared to Carina-Sagittarius and Perseus. Another basic parameter is the epicyclic frequency (the residual circular velocity after subtracting the Galactic rotation curve) which Lépine et al. (2008) find to be $42 \pm 4 \mathrm{~km} \mathrm{~s}^{-1} \mathrm{kpc}^{-1}$ at the solar radius. 


\section{Formation and evolution}

The ages of the oldest OCs place a lower limit on the age of the Galactic disk. Presently, the oldest-known clusters are (still) NGC 6791 and Be 17 which have ages of around 10 Gyr (Carraro et al. 1999; Salaris et al. 2004), which is younger than the halo, although they almost fall in the range of the youngest globular clusters.

Few old open clusters have been identified in the inner Galaxy. This is usually attributed to the harsher conditions that would destroy OCs at small Galactocentric radii. Curiously, the orbit of NGC 6791, which is one of the oldest known OCs and is presently outside the solar circle, can reach a distance of only $4.6 \mathrm{kpc}$ from the Galactic Centre.

The most discussed aspects of Galactic evolution scenarios are those related to chemical-abundance gradients. Most observational studies in the last two decades find a smoothly decreasing metallicity-Galactic radius gradient. Recent studies determine a gradient of $\sim-0.06 \mathrm{dex} \mathrm{kpc}^{-1}$ (Friel et al. 2002; Chen et al. 2003). However, controversy has been generated by the work of Twarog et al. (1997), who reexamined the metallicity scales of published observations and found the radial metallicity distribution to be better described by a step function with the transition occurring at around $10 \mathrm{kpc}$ from the Galactic Centre. The inner region would be characterised by a shallow gradient (with an average $[\mathrm{Fe} / \mathrm{H}]=0.0 \mathrm{dex}$ ), followed by a -0.3 dex step and then an almost flat metallicity distribution. On the theoretical side, models usually yield simple gradients, although the recent paper of Magrini et al. (2009) is able to predict a sudden change at around $7 \mathrm{kpc}$, followed by an almost constant plateau starting at around $11 \mathrm{kpc}$. From a dynamical point of view, the simulations of Corder \& Twarog (2001) show how orbital diffusion can smooth the discontinuity (assuming it is real) and how using younger clusters enhances the detection probability of the step. Another perspective is offered by Mishurov et al. (2002) and Lépine et al. (2003) who show how the co-rotation resonance can break a smooth abundance gradient.

Regarding a possible age-metallicity relation, most studies fail to find such a dependence over a cluster age range of almost $10 \mathrm{Gyr}$, although there is marginal evidence from clusters at Galactocentric radii larger that $10 \mathrm{kpc}$ (see Friel 1995; Friel et al. 2002). The most natural explanation remains that chemical enrichment must have essentially taken place early in the Galaxy's evolution.

For a more in-depth view of OCs as probes of the origin and chemical evolution of the Milky Way the reader is referred to the reviews of Janes \& Phelps (1994), Friel (1995) and Friel et al. (2002).

\section{Interstellar extinction}

We now briefly return to the point from which this review started: extinction. Joshi (2005) studied the interstellar-absorption pattern within $\sim 4-5 \mathrm{kpc}$ using catalogued reddening values towards 722 OCs. He found that absorption approximately follows a sinusoidal curve with Galactic longitude, with the highest values occurring at $l \sim 30$ $50^{\circ}$ and the lowest values around $l \sim 220-250^{\circ}$. Extinction is generally low in the $3 \mathrm{GQ}$,

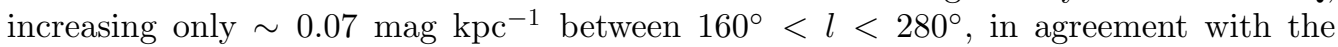
analysis by Vázquez et al. (2008). Except for the opaque barrier at $l \sim 265^{\circ}$ due to the Vela molecular ridge, the low-absorption window continues in the 4GQ towards Carina. In general, for the remaining longitude range, absorption is higher and happens mostly between 1 and $2 \mathrm{kpc}$ from the Sun.

Joshi (2005) also determined that the dust plane is tilted by $0.6^{\circ}$ with respect to the $b=0^{\circ}$ plane. The maximum and minimum height of the dust plane occur at $\sim 54$ and $234^{\circ}$, respectively, coincident with the maximum and minimum of the Galactic warp. 
Although these and other studies reveal the distribution and amount of interstellar matter, they do not give any idea of the nature of the absorbing material. This is due to most $\mathrm{OC}$ reddening and absorption values having been derived assuming a standard interstellar extinction law. A study of the variations of the interstellar law would certainly be interesting.

\section{Prospects}

As insistently advocated in this contribution, an important goal for the future is to have an unbiased, or at least well-understood, sample of OCs. This will require mitigating extinction artifacts, which demands analysing the mostly unstudied infrared clusters. On the way, we will learn much about the kind, quantity and distribution of interstellar material. Success will, however, depend on the development of IR photometric analysis techniques capable of yielding precise cluster parameters.

Another well-known problem to be addressed is that of the homogeneity of OCparameter compilations. Homogeneity of the data itself is also certainly an issue, but of second order when compared to the analysis. However, a word of caution should be issued: homogeneous methods do not necessarily produce homogeneous catalogues. As an example, Hipparcos parallaxes are great for determining the distance to close clusters such as the Pleiades. For a cluster at 300 pc, parallaxes will still be available but will not do a very good job. In this case, a good ZAMS fit would produce a distance on a scale in better agreement with the Pleiades distance from parallaxes.

As for the future, one cannot emphasise enough how important Gaia will be to Galactic astronomy. Gaia will deliver excellent distance estimates and kinematics for single stars, but it will not end the use of OCs for studying the Galaxy. Clusters are still unique when we need ages, and Gaia will help improving them. Also, Gaia is not an IR mission and will therefore not be able to penetrate dense regions.

Many large-area surveys are either ongoing or planned for the near future using groundbased facilities: UKIDSs, SEGUE, IPHAS, VPHAS+, VVV, VHS, LSST, PAN-STARRS and others. These surveys will produce a wealth of data from the optical to the near-IR, including in selected bands such as $\mathrm{H} \alpha$. In several cases, observations will even be timeresolved.

The next few years promise to offer a profusion of intense emotions to OC researchers.

\section{Acknowledgements}

Although I appear as the sole author of this contribution, it has also been the product of years of stimulating discussions with several colleagues. I am particularly grateful to Wilton Dias, Giovanni Carraro, Rubén Vázquez and Jacques Lépine. This work was partially supported under a bilateral FCT/CAPES agreement.

\section{References}

Alessi, B. S., Moitinho, A., Dias, W. S., \& Lépine, J. R. D. 2003, A\& A, 410, 565

Becker, W. \& Fenkart, R. B. 1970, in: W. Becker \& G. I. Kontopoulos (eds.), The Spiral Structure of our Galaxy, IAU Symp. (Dordrecht: Reidel) vol. 38, p. 205

Bica, E., Dutra, C. M., \& Barbuy, B. 2003a, A\&\&A, 397, 177

Bica, E., Dutra, C. M., Soares, J., \& Barbuy, B. 2003b, A\& $A, 404,223$

Bobylev, V. V., Bajkova, A. T., \& Lebedeva, S. V. 2007, As. Lett., 33, 720

Bonatto, C., Kerber, L. O., Bica, E., \& Santiago, B. X. 2006, A\&SA, 446, 121

Carraro, G., Girardi, L., \& Chiosi, C. 1999, MNRAS, 309, 430 
Carraro, G., Vázquez, R. A., Costa, E., Perren, G., \& Moitinho, A. 2009, ApJ, submitted

Carraro, G., Vázquez, R. A., \& Moitinho, A. 2008, A\&A, 482, 777

Carraro, G., Vázquez, R. A., Moitinho, A., \& Baume, G. 2005, ApJ (Letters), 630, L153

Chen, L., Hou, J. L., \& Wang, J. J. 2003, AJ, 125, 1397

Corder, S. \& Twarog, B. A. 2001, AJ, 122, 895

Cutri, R. M., et al. 2003, explanatory Supplement to the 2MASs All Sky Data Release

Dias, W. S., Alessi, B. S., Moitinho, A., \& Lépine, J. R. D. 2002, A\& A, 389, 871

Dias, W. S. \& Lépine, J. R. D. 2005, ApJ, 629, 825

Dutra, C. M., Bica, E., Soares, J., \& Barbuy, B. 2003, A\&A A, 400, 533

Eisenhauer, F., Schödel, R., Genzel, R., Ott, T., Tecza, M., Abuter, R., Eckart, A., \& Alexander, T. 2003, ApJ (Letters), 597, L121

ESA 1997, The Hipparcos and Tycho Catalogues (European Space Agency)

Friel, E. D. 1995, ARA $\& A, 33,381$

Friel, E. D., Janes, K. A., Tavarez, M., Scott, J., Katsanis, R., Lotz, J., Hong, L., \& Miller, N. 2002, AJ, 124, 2693

Frinchaboy, P. M. \& Majewski, S. R. 2008, AJ, 136, 118

Froebrich, D., Scholz, A., \& Raftery, C. L. 2007, MNRAS, 374, 399

Genzel, R., Pichon, C., Eckart, A., Gerhard, O. E., \& Ott, T. 2000, MNRAS, 317, 348

Girardi, L., Bressan, A., Bertelli, G., \& Chiosi, C. 2000, A\&AS, 141, 371

Glushkova, E., Koposov, S., Zolotukhin, I., Beletsky, Y., Vlasov, A., \& Leonova, S. 2009, Ast. Lett., in press (arXiv:0910.1330)

Høg, E., Fabricius, C., Makarov, V. V., Urban, S., Corbin, T., Wycoff, G., Bastian, U., Schwekendiek, P., \& Wicenec, A. 2000, A\&A (Letters), 355, L27

Janes, K. A. \& Adler, D. 1982, ApJS, 49, 425

Janes, K. A. \& Phelps, R. L. 1994, AJ, 108, 1773

Janes, K. A., Tilley, C., \& Lyngå, G. 1988, AJ, 95, 771

Joshi, Y. C. 2005, MNRAS, 362, 1259

Joshi, Y. C. 2007, MNRAS, 378, 768

Kharchenko, N. V., Piskunov, A. E., Röser, S., Schilbach, E., \& Scholz, R. 2005, A\& A, 440, 403 Kroupa, P. 2002, MNRAS, 330, 707

Lépine, J. R. D., Acharova, I. A., \& Mishurov, Y. N. 2003, ApJ, 589, 210

Lépine, J. R. D., Dias, W. S., \& Mishurov, Y. 2008, MNRAS, 386, 2081

Lyngå, G. 1987, Computer-based Catalogue of Open Cluster Data, 5th ed., Tech. rep., Observatoire de Strasbourg, Centre de Données Stellaires, Strasbourg

Magrini, L., Sestito, P., Randich, S., \& Galli, D. 2009, A\&A, 494, 95

Martínez-Delgado, D., Butler, D. J., Rix, H.-W., et al. 2005, ApJ, 633, 205

Mermilliod, J. C. 1992, Bull. Inform. CDS, 40, 115

Mishurov, Y. N., Lépine, J. R. D., \& Acharova, I. A. 2002, ApJ (Letters), 571, L113

Moffat, A. F. J., Jackson, P. D., \& Fitzgerald, M. P. 1979, A\& AS, 38, 197

Moitinho, A., Vázquez, R. A., Carraro, G., Baume, G., Giorgi, E. E., \& Lyra, W. 2006, MNRAS (Letters), 368, L77

Piskunov, A. E., Kharchenko, N. V., Röser, S., Schilbach, E., \& Scholz, R. 2006, A\&4A, 445, 545

Platais, I., Kozhurina-Platais, V., \& van Leeuwen, F. 1998, AJ, 116, 2423

Salaris, M., Weiss, A., \& Percival, S. M. 2004, A\&A, 414, 163

Shen, M. \& Zhu, Z. 2007, ChJA\&A, 7, 120

Stetson, P. B., Davis, L. E., \& Crabtree, D. R. 1990, in: G. H. Jacoby (ed.), CCDs in astronomy, ASP Conf. Ser. (San Francisco: ASP), vol 8, p. 289

Trumpler, R. J. 1930, PASP, 42, 214

Twarog, B. A., Ashman, K. M., \& Anthony-Twarog, B. J. 1997, AJ, 114, 2256

Vázquez, R. A., May, J., Carraro, G., Bronfman, L., Moitinho, A., \& Baume, G. 2008, ApJ, 672,930

Zacharias, N., Urban, S. E., Zacharias, M. I., Wycoff, G. L., Hall, D. M., Monet, D. G., \& Rafferty, T. J. 2004, AJ, 127, 3043 